\title{
Welcome copernicium?
}

In the search for superheavy elements, element 112 was a stepping stone towards the 'islands of stability'. Sigurd Hofmann now relates the steps that led to its 'creation' and detection.

E lement 112 did not invite a lot of interest when theoretical nuclear physicists predicted the famous island of stable superheavy elements - elements that have a number of nucleons that leads to arrangements in closed shells, which confers them stability despite the high repulsion forces between the protons. Rather than element 112 , it was element 126 , and later 114 and 120, that attracted most of the attention, because they were predicted to have half-lives up to a million years, suggesting that they could be found on Earth. Efforts to identify them in nature or in various nuclear reactions, however, remained unsuccessful, leading scientists to follow the difficult and stony path of producing these elements step-by-step. In 1976, the starting point was element 106 , now called seaborgium, and for many years element 112 marked a temporary end to this progress.

Four key technical improvements were required to enable the detection of 112 . The first was an accelerator delivering ion beams of as many different isotopes as possible, with beam intensities of at least $10^{12}-10^{13}$ ions per second, and at about $10 \%$ of the speed of light. The second was a target, also made from various isotopes, which could stand these high beam intensities. The third was a separator for fast and efficient separation of the reaction products from the beam, and finally a detector system for a reliable identification of the element obtained.

In our laboratory, these advances were made possible with the accelerator UNILAC (Universal Linear Accelerator), a rotating target wheel, the electromagnetic separator SHIP (Separator for Heavy Ion reaction Products) and the use of position-sensitive silicon detectors. This combination allowed us to detect nuclei with lifetimes ranging from one microsecond to a few hours, and detect each element from 107 to 111. In 1996 we were ready to synthesize element 112. We selected a beam and a target

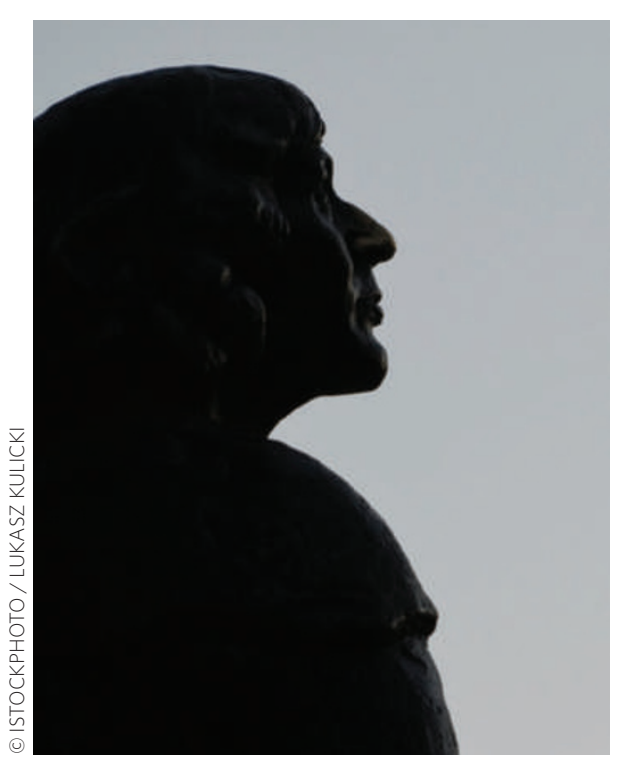

Nicolaus Copernicus is best known for challenging the accepted view of the Universe by describing a system centred around the Sun, rather than the Earth.

material so that the sum of their protons gave 112: a zinc beam consisting of 30 protons and 40 neutrons was used to bombard lead target nuclei, composed of 82 protons and 126 neutrons, resulting in a new element that had 112 protons and 166 neutrons - that is, an atomic-mass number of 278 .

Although forcing the two nuclei (zinc and lead) together requires a considerable amount of energy - the reacting nuclei do not easily amalgamate, and are much more likely to be repulsed by immensely strong electric forces acting between their protons - this barrier can be overcome and the attractive force between the nuclei leads to nuclear fusion. This reaction releases energy, and the hot nucleus created by this exothermic process then cools down by emitting one neutron, and the nucleus 277 remains, which is the one we were able to study.

Even with the most intensive beams, we managed to produce only one atom of element 112 in one week. In 2000, a second experiment allowed us to measure a second atom, and two more atoms were produced in 2004 by the RIKEN laboratory in Japan (K. Morita et al. J. Phys. Soc. Jpn. 76, 043201; 2007), thus confirming our data. This led the International Union of Pure and Applied Chemistry to assign priority of its discovery to our team, and in April 2009 we were asked to suggest a name for element 112 .

Our team of 21 researchers from four different nations discussed possible names for about a month - including those suggested by students and the public. We agreed that element 112 should be named after the astronomer Nicolaus Copernicus and suggested the name 'copernicium' with the abbreviation ' $\mathrm{Cn}$ '.

Copernicus, who lived 500 years ago in the transition period between the Middle Ages and modern times, had a significant influence on the political and philosophical thinking at that time and contributed to the rise of modern science, based on experiments. His representation of the planets also represents other systems governed by an attractive force between small objects revolving around a bigger centre. On a microscopic scale, this is the atom, with its electrons orbiting around the nucleus and, in the case of copernicium, 112 electrons moving around a nucleus consisting of 112 protons and 165 neutrons.

Copernicium should behave as a transition metal, as it is below zinc, cadmium and mercury in group 12, and its similarity to the latter has already been shown by initial experiments adsorbing a few atoms of it onto a gold surface. It might be a little bit more volatile than mercury, but will very likely be a liquid at room temperature. Of course, with only a few rapidly decaying atoms having been made so far, it is not likely to be of practical use for some time, but its detection paves the way to heavier elements still — the aforementioned superheavy elements.

SIGURD HOFMANN is at the Heavy lon

Research Centre (GSI) in Darmstadt, Germany.e-mail: s.hofmann@gsi.de
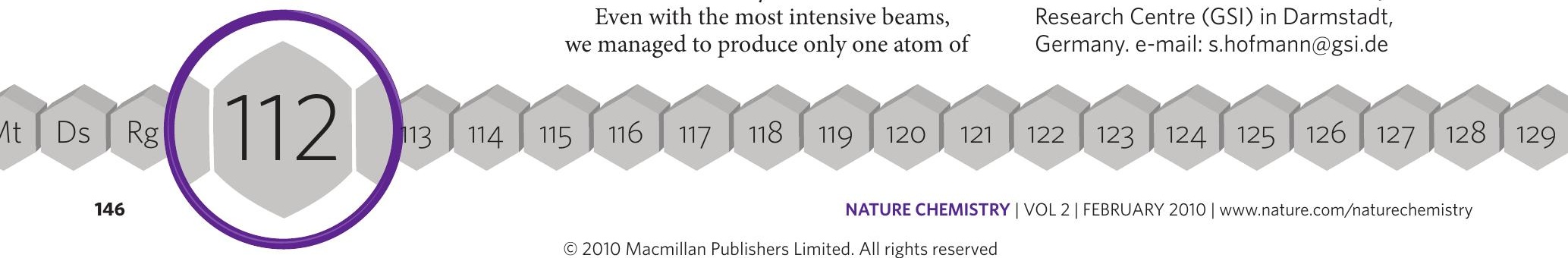